\title{
Computational hemodynamics analysis in realistic $3 D$ geometries of the human coronary atherosclerosis
}

\author{
S. I. Bernad ${ }^{1}$, T. Bărbat ${ }^{1}$, E. Bernad ${ }^{2}$ \& R. Susan-Resiga ${ }^{3}$ \\ ${ }^{1}$ Romanian Academy - Timisoara Branch, Timisoara, Romania \\ ${ }^{2}$ University of Medicine and Pharmacy Timisoara, Timisoara, Romania \\ 3 "Politehnica" University of Timisoara, Timisoara, Romania
}

\begin{abstract}
The present investigation concentrates on one particular problem related to fluid mechanics: namely, the description of disturbed flow fields in the coronary atherosclerosis. Atherosclerosis creating a constriction can significantly alter the local blood flow dynamics. From a biological aspect, the changes that take place in the flow have a profound effect on the structure and function of the arterial wall and the development of the disease. The purpose of this paper was to non-invasively assess hemodynamic parameters, such as mass flow, wall shear stress, and wall pressure, with computational fluid dynamics (CFD) in coronary arteriosclerosis using patient-specific data from computed tomography (CT) angiography.
\end{abstract}

Keywords: blood flow, coronary artery, hemodynamics, numerical simulation.

\section{Introduction}

Statistics show that atherosclerosis is the leading cause of death in European countries. The vessels most commonly affected are the abdominal aorta, femoral, carotid, and coronary arteries.

A number of reports indicate that arterial stenosis has a significant effect on the character of blood flow in blood vessels. For example, stenosis induces formation of local vortices, increases energy losses, changes the pressure on the blood vessel walls, and modifies flow parameters in the branching distal portions of the vessels [4, 7-9]. Blood flowing through a vascular segment exerts a 
tangentially directed shear stress on the luminal surface of endothelial cells, the inner layer of the vascular wall. Wall shear stress is the product of wall shear rate and local blood viscosity. Wall shear stress has been shown to be an important determinant of endothelial cell function $[3,4,6]$.

Atherosclerosis involves the development of plaque in the inner lining of large and medium sized arteries. Eventually the plaque matures into a structure consisting of two main parts: a soft "inner core" that consists of cholesterol, waste products, inflammatory cells and calcium, and a thin outer shell called the "fibrous cap" [6]. As plaque builds up, it can begin to block the flow of blood through an artery.

Hemodynamic flow assessment in coronary arteries is usually performed with intravascular Doppler ultrasound by measuring local velocities [1, 5]. Even if these data enable a hemodynamic characterization of stenosis severity, the introduction of an ultrasound-catheter into the lumen is an invasive procedure, leads to flow disturbances, and the results of such measurements are, therefore, often difficult to interpret [5]. An alternative means for invasive flow measurements is presented by the calculation of models in which blood flow can be virtually simulated, a method that is called computational fluid dynamics (CFD). In fact, several in vitro studies [3, 6] and some in vivo investigations [11] have shown that CFD allows reliable physiologic blood flow simulation and measurements of WSS, wall pressure, and mass flow. A requisite for obtaining reliable results from coronary $\mathrm{CFD}$ is to use exact anatomical models [7, 9], which today are provided by multi-detector row computed tomography (CT). The three-dimensional reconstruction of coronary arteries from CT datasets has been shown to be more accurate than corresponding 3D reconstructions obtained from conventional angiography combining two simultaneously captured twodimensional views $[1,2,6]$. The purpose of this study was to simulate pulsatile blood flow in coronary arteries using CFD based on geometric models from CT datasets and to measure the WSS, wall pressure, and mass flow and visualize flow patterns.

\section{Materials and method}

For the case presented in this paper, spiral CT (computed tomography) was performed 4 days following the CA (coronary angiography), (44 year old, patient by typical angina symptoms is investigated). A Somatom Sensation 64 Scanner (Siemens Medical Systems, Erlangen, Germany) was used in non-enhanced spiral scan technique with a slice thickness of $2 \mathrm{~mm}$, a table feed of $3 \mathrm{~mm} / \mathrm{s}$, and an increment of $2 \mathrm{~mm}$. Data corresponding to the investigated patient is presented in Table 1.

The Vascular Model: the coronary artery model simulating the flow field in the RCA (right coronary artery) is illustrated in fig. 1 and fig. 2 respectively. The RCA is modelled to be of length $52 \mathrm{~mm}$ with variable diameters, depending of the stenosis severity. Model assumptions, data input, and boundary conditions: the blood is assumed to be incompressible, with a Newtonian behaviour having dynamic viscosity $(\mu)$ of $0.00408 \mathrm{~Pa}$ and a density $(\rho)$ of $1050 \mathrm{~kg} / \mathrm{m} 3$. 
Table 1: $\quad$ Preoperative patient characteristics.

\begin{tabular}{lc}
\hline Variables & Value \\
\hline Mean age & 44 \\
Gender (M/F) & male \\
History of MI & $\mathrm{NO}$ \\
Previous PTCA & $\mathrm{NO}$ \\
Renal insufficiency & $\mathrm{NO}$ \\
Cardiovascular risk factor & \multicolumn{2}{c}{ Yes } \\
Hypertension & $\mathrm{NO}$ \\
Diabetes & Yes \\
Smoke & moderate \\
Obesity & multiple/severe \\
Angiographic data & intermediate \\
RCA stenosis & \\
LCX stenosis & \multicolumn{2}{c}{ coronary angioplasty. } \\
\hline MI = myocardial infarction, PTCA = percutaneous transluminal \\
RCA = right coronary artery, LCX = left circumflex artery.
\end{tabular}

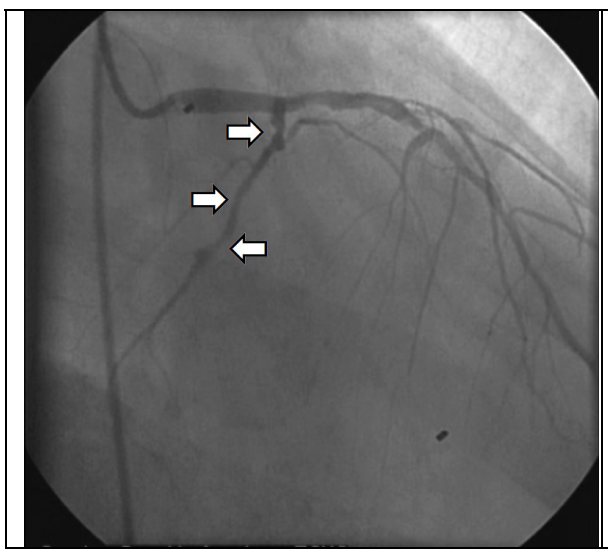

Figure 1: Invasive coronary angiography (CA). Multiple stenosis of right coronary artery (arrows).

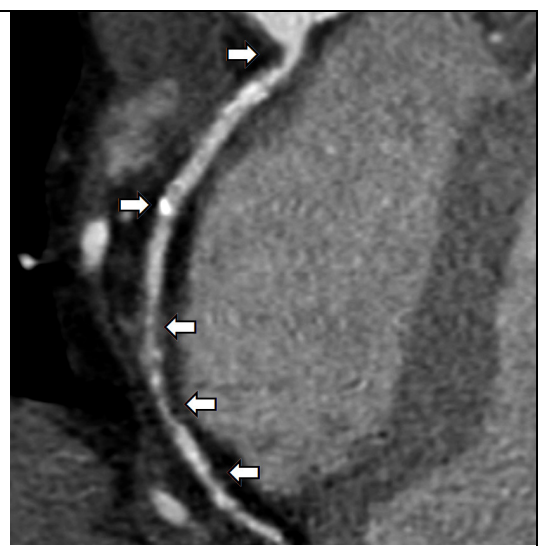

Figure 2: Axial tomographic images of heart after intravenous injection of contrast agent (64slice CT).

The blood vessel walls are assumed to be rigid and impermeable. The distributions of velocity and wall shear stress are obtained by computationally solving the Navier-Stokes equations.

\subsection{Flow parameters}

To complement the geometric factors we must also consider the physiological flow parameters that describe our problem. A wide variety of pulsatile flow 
waveforms are observed in arteries, these vary with position in the arterial system, in response to exertion, and from individual to individual. We assume that $\bar{u}(t)$ is described by the mean and two harmonic terms in the form [10]:

$$
\bar{u}(t)=\bar{u}_{m}\left[1+a_{1} \sin \left(\frac{2 \pi t}{T}\right)+a_{2} \cos \left(\frac{4 \pi t}{T}\right)\right]
$$

The pulsatile flow waveforms presented in eqn. (1), is more realistic approximation of physiologic waveforms since it has a higher peak-to-mean ratio, is obtained using two harmonics and with $a 1=-a 2=0.75$. We note that if the magnitude of the two harmonics is fixed at 0.75 , fig. 3 .

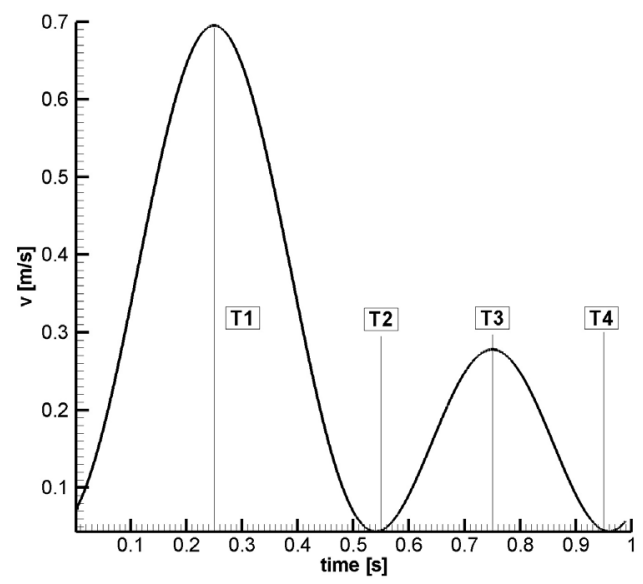

Figure 3: Waveforms of $\bar{u}(t)$ in the pulsatile flow cases considered, eqn. (1).

Fluid dynamics simulation setup: the fluid dynamics simulations are performed by using a control-volume-based technique, implemented in the computational fluid dynamics code, FLUENT [11]. The computation procedure of the commercial code consists of (i) construction of the geometry using a preprocessor, GAMBIT [11], (ii) meshing the computation domain, (iii) assigning boundary conditions in terms of velocities and flow-rate weightings, (iv) assigning fluid properties, and (v) the solution algorithm.

The geometry of the stenosed coronary artery is constructed using the dimensions provided by the CT measurements. In figures $4 \mathrm{c}$ the reconstructed coronary artery is shown to have a circular cross-section, larger distal to the stenosed area.

In order to carry out the mesh sensitivity analysis, numerical simulations were carried out by varying the number of mesh elements in the computational domain. The accuracy of the simulation results was then improved by employing a finer mesh that contained 1598752 elements. 


\section{Results and discussion}

In order to generate the geometry for the present study, a series of stenosed right coronary artery (RCA) slices were acquired in vivo using CT scan imaging. A profile of the reconstructed RCA is shown in fig. 4A. A very important aspect of the analysis of three-dimensional flows in the RCA is the graphical presentation of the flow field.

The vortical type velocity field has been induced by the curved entrance geometry, and it is not a result of the inlet condition, fig. 5.

RCA curvature gradually changes the flow in sections $d_{0}, d_{1}, d_{2}, d_{3}$. Far downstream of the section $d_{3}$, the flow is highly three-dimensional with little resemblance to classical steady flow in a pipe, being sharply skewed toward the outer wall of the RCA.

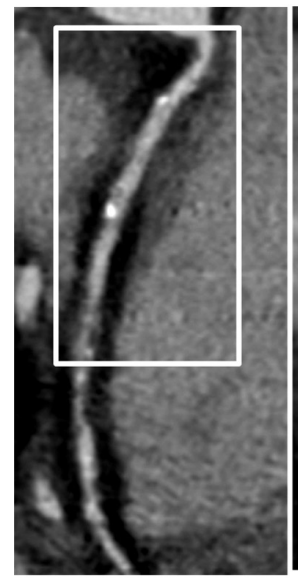

A

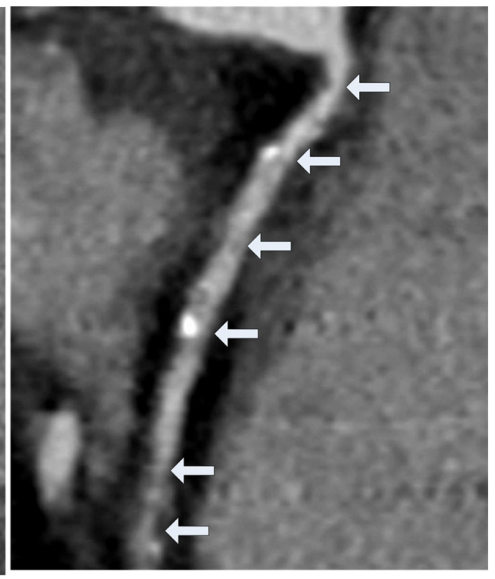

B

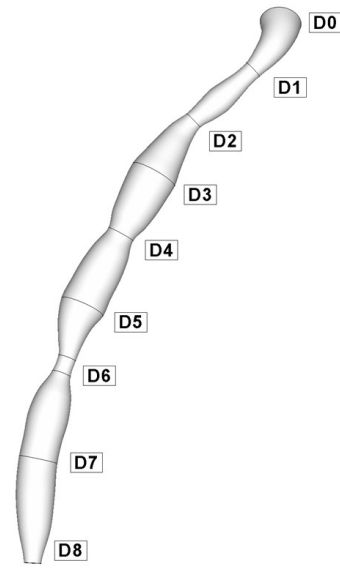

C

Figure 4: Axial tomographic images of the RCA (right coronary artery), 64slice CT (A); multiple stenosis of right coronary artery (arrows), (B); 3-D geometry reconstruction (C).

The natural curvature of the RCA is expected to induce significant secondary flow motion or crossflow motion within the artery, and the presence of the successive curvature has an impact on the structure of the induced secondary flows.

Figure 5 depicts the distribution of velocity-vectors in the flow field for the different time step. In section $\mathrm{d}_{0}$, the inner wall of the RCA curvature creates a recirculation region.

In constricted regions of the RCA between the sections $d_{1}$ and $d_{6}$ a strong region of recirculation is observed near to the inner wall, which forces the flow to move distal in the bypass graft, figures 5 and 7 . The average velocity and pressure distribution in the different sections of the bypass graft is presented in Table 2. 
Table 2: Hemodynamic data in different sections of the venous bypass graft.

\begin{tabular}{|c|c|c|c|c|c|c|c|c|}
\hline \multirow{2}{*}{ Section } & \multicolumn{2}{|c|}{ Time T1 } & \multicolumn{2}{c|}{ Time T2 } & \multicolumn{2}{c|}{ Time T3 } & \multicolumn{2}{c|}{ Time T4 } \\
\cline { 2 - 9 } & ${ }^{*} V_{T 1}$ & ${ }^{* *} P_{T 1}$ & ${ }^{*} V_{T 2}$ & ${ }^{* *} P_{T 2}$ & ${ }^{*} V_{T 3}$ & ${ }^{* *} P_{T 3}$ & ${ }^{*} V_{T 4}$ & ${ }^{* *} P_{T 4}$ \\
\hline D0 & 0.15 & 28.2 & 0.0096 & 1.40 & 0.06 & 8.78 & 0.0096 & 0.88 \\
\hline D1 & 0.69 & 25.4 & 0.0447 & 1.36 & 0.277 & 8.20 & 0.0447 & 0.85 \\
\hline D2 & 0.66 & 23.8 & 0.0429 & 1.29 & 0.266 & 7.75 & 0.0429 & 0.82 \\
\hline D3 & 0.15 & 24.4 & 0.0125 & 1.28 & 0.055 & 7.85 & 0.0140 & 0.81 \\
\hline D4 & 0.32 & 24.2 & 0.0209 & 1.27 & 0.128 & 7.79 & 0.0209 & 0.82 \\
\hline D5 & 0.12 & 24.4 & 0.0111 & 1.26 & 0.049 & 7.80 & 0.0083 & 0.82 \\
\hline D6 & 0.76 & 21.1 & 0.0496 & 1.22 & 0.307 & 7.13 & 0.0496 & 0.82 \\
\hline D7 & 0.68 & 20.2 & 0.0441 & 1.19 & 0.273 & 6.86 & 0.0441 & 0.79 \\
\hline D8 & 0.21 & 21.1 & 0.0147 & 1.17 & 0.069 & 7.05 & 0.0151 & 0.77 \\
\hline D9 & 0.84 & 17.5 & 0.0547 & 1.12 & 0.330 & 6.26 & 0.0547 & 0.77 \\
\hline
\end{tabular}

*Area Weighted Average Velocity Magnitude [m/s] for time steps T1, T2, T3, T4.

**Area Weighted Average Pressure [mmHg] for time steps T1, T2, T3, T4.

For the patient of this study, the wall pressure decreased towards the periphery of the coronary artery tree with elevated pressure drops in stenotic segments. The increased pressure drop in stenoses reflects the elevated energy needed to drive the flow through these regions.

As shown in atherosclerotic coronary arteries, regions of flow acceleration were associated with high WSS. Relating our CFD results to coronary artery morphology, the location of atherosclerotic plaques correlated well with the regions of high WSS.

The flow pattern variations give us insight into the wall shear distribution. The high velocity gradients in the anastomosis give rise to large spatial variations in the resulting wall shear stress.

In the spacing between successive constrictions (sections $d_{1}$ and $d_{6}$ ) the flow repeatedly undergoes an expansion after each constriction where separation zone formed distal to the section $\mathrm{d}_{2}, \mathrm{~d}_{4}$, and $\mathrm{d}_{6}$, and re-attaches on the wall between the two constrictions. This separation zone subsequently covers the whole space between the two constrictions and leads to vortex shedding.

Secondary flows subtract energy from the forward axial flow motion. Thus their location and extent can affect severely the fluid dynamic performances in stenosed artery.

Wall shear stress and pressure are likely the most relevant parameters from the standpoint of the fluid mechanical involvement in the development of atherosclerosis.

The wall shear stress distribution patterns shown in fig. 6 suggest that the maximum shear stresses occur in the vicinity of the stenosed regions. Parallel to the changes of WSS, the flow pattern was more variable and inconsistent in vicinity of the constriction. 


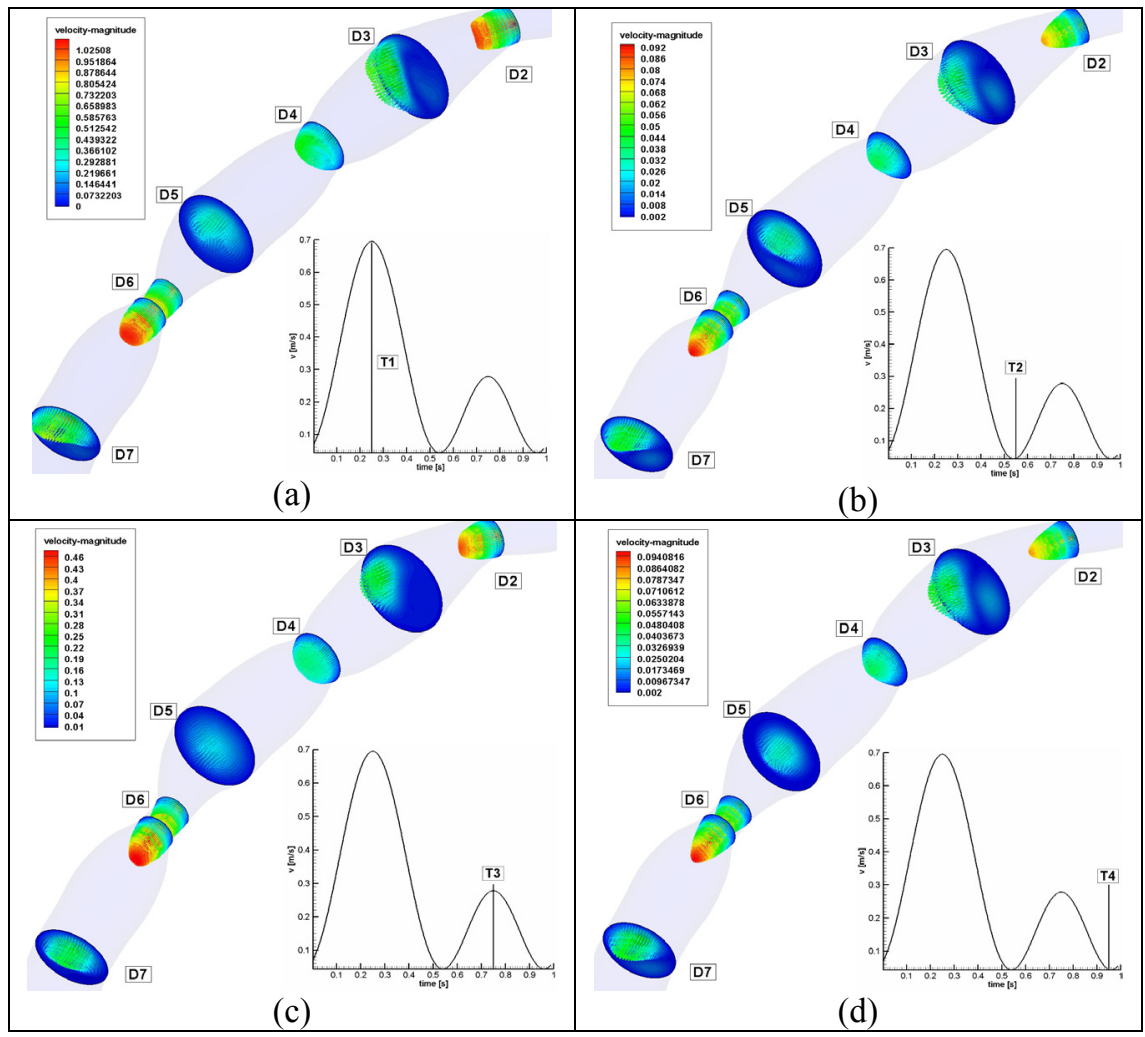

Figure 5: Velocity field and vector computed in the stenosed RCA. Parabolic profiles of the velocity vectors are seen inside the RCA. Maximum flow velocity approaching the artery curvature in sections $\mathrm{d}_{6}$ (maximum constriction) is around $1 \mathrm{~m} / \mathrm{s}$. The flow exiting the RCA curvature in section $d_{2}$ with a higher velocity results in a stronger impingement on the wall of the artery. The maximum flow velocity magnitude $\left(1 \mathrm{~m} / \mathrm{s}\right.$ in section $\mathrm{d}_{6}$ and $0.8 \mathrm{~m} / \mathrm{s}$ in section $\left.\mathrm{d}_{2}\right)$, seen close to the exterior wall in section $\mathrm{d}_{2}$, and $\mathrm{d}_{6}$.

Artery section constriction and post-constriction dilatations have led to acceleration and rapid deceleration, respectively, including a distortion of flow. Large recirculation regions found in the vicinity of the each constricted section.

An effective way to investigate these highly three-dimensional fluid motions is to numerically inject a passive or non-interactive tracer into the flow, and we have carried out such an investigation in the present work. We have introduced a tracer, which serves as a visualization tool to analyze the flow characteristics.

Flow separation occurs on the inner walls. However, the separation zone on the inner wall downstream curvature of the section $\mathrm{d}_{2}$ is larger that separation zone downstream of the section $\mathrm{d}_{4}$, fig. 7 . Prominent vortex shedding with $3 \mathrm{D}$ features is seen at the end of the flow deceleration zone in the spacing between the constrictions. 


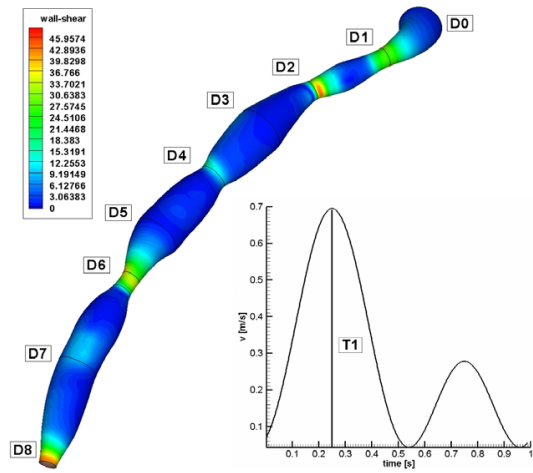

(a)

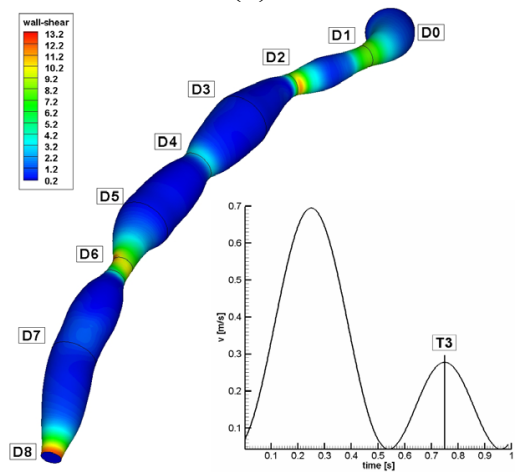

(c)

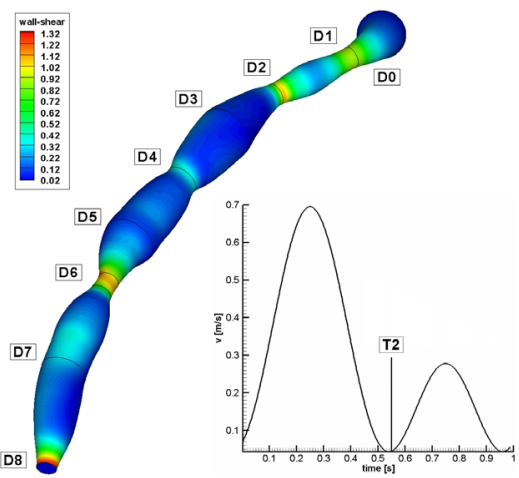

(b)

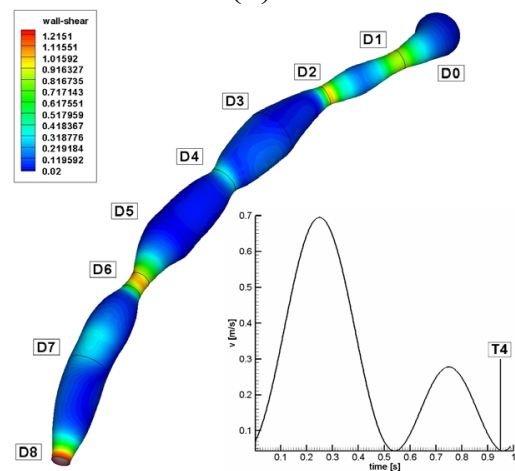

(d)

Figure 6: Wall shear stress distribution on the RCA wall for different time steps (fig. 3). There are many regions of elevated wall shear stress correlating either to artery curvature or to irregular vessel geometry (stenosed regions).

Figure 7 present the tracer distribution inside to the stenosed right coronary artery for different time steps. The associated velocity profiles are shown in fig. 5. However, in the low-velocity regions, i.e., distal to the sections $\mathrm{d}_{2}, \mathrm{~d}_{4}$, and $\mathrm{d}_{6}$, the relatively long residence times for particles help them to establish a relatively large mass-transfer zone.

The role of secondary flows in particle transport is not as pronounced as it is for micro particles. Specifically, in some cases micro particles can be swept out of the strong secondary vortices due to their inertia, and preferential ring areas of high concentrations are generated outside the secondary vortices.

\section{Conclusions}

The problem of flow disturbances in the arterial system is generally complex from the fluid dynamics point of view. For physicians, three-dimensional 


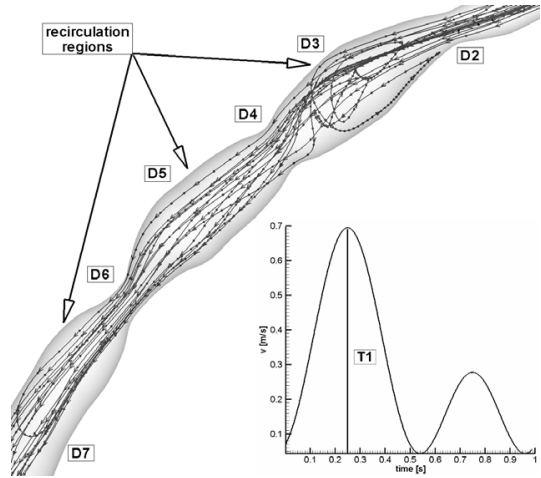

(a)

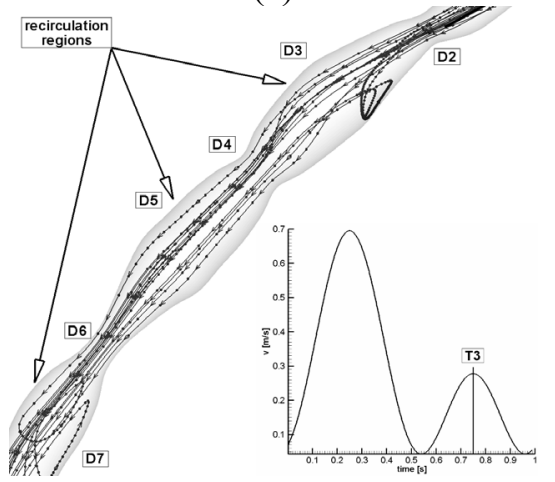

(c)

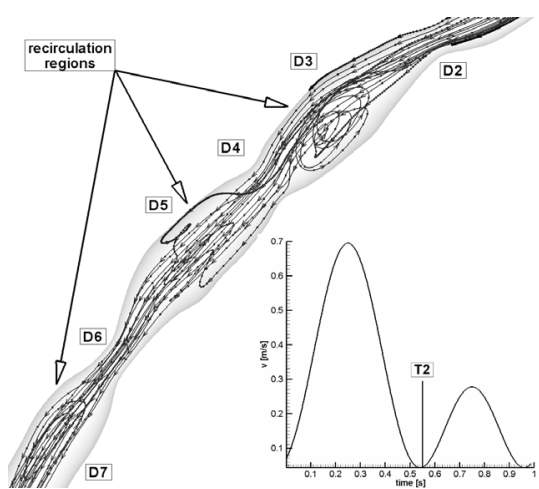

(b)

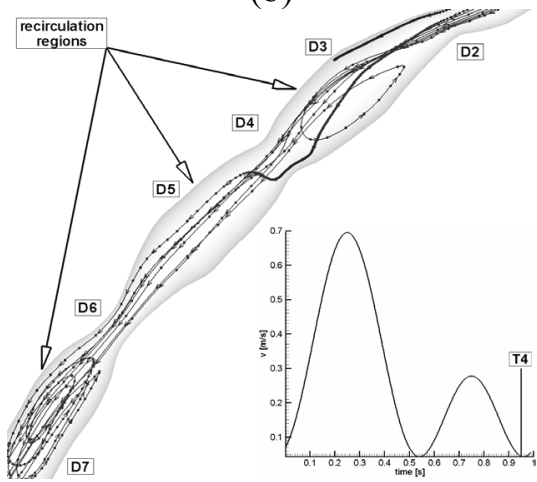

(d)

Figure 7: Particles motion inside the stenosed coronary artery (RCA).

modelling becomes particularly helpful when dealing with complex vascular geometries as seen with large stenosis.

Highly accurate anatomy for the generation of geometric models is a principal requirement to perform reliable flow simulations and to make assumptions about mass flow, WSS, and wall pressure.

In summary, this paper describes a method for the construction of a flow model based on two-dimensional in vivo images acquired noninvasively. In order to create a real physical artery model, the method uses commercially available software, both to create an anatomy model and reproduce the blood hemodynamics. In conclusion, our study demonstrates that the simulation of pulsatile blood flow is feasible in-vivo in coronary arteries of patients with geometric data obtained from multi-detector row CT.

\section{Acknowledgements}

The present research has been supported by the Romanian National Authority for Scientific Research through the CNCSIS 798/2008 project. 


\section{References}

[1] Achenbach S., Current and future status on cardiac computed tomography imaging for diagnosis and risk stratification, Journal of Nuclear Cardiology, 12(6), pp: 703-713, 2005.

[2] Bernad S.I., Barbat T., Bernad E.S., Susan-Resiga R., Cardio vascular surgery - simulation based medical intervention, Proceedings of the 9th WSEAS Int. Conf. on MATHEMATICS \& COMPUTERS IN BIOLOGY \& CHEMISTRY (MCBC '08), eds. L Vladareanu, V. Chiroiu, P. Bratu, I. Magheti, WSEAS Press, pp: 100-106, 2008.

[3] Gardhagen R., Renner J., Lanne T., Karlsson M., Subject Specific Wall Shear Stress in the Human Thoracic Aorta, WSEAS TRANSACTIONS on BIOLOGY and BIOMEDICINE, 10(3), pp: 609-614, 2006.

[4] Hornero F., Cervera V., Estornell J., Rodirguez I., Buendia J.A., Esteban J.M., Montero J.A., Virtual Vascular Endoscopy for Acute Aortic Dissection, Ann Thorac Surg, 80, pp: 708-710, 2005.

[5] Ruengsakulrach P., Joshi A.K., Fremes S., Foster S., Wall Shear Stress and Atherosclerosis: Numerical Blood Flow Simulations in the Mouse Aortic Arch, WSEAS TRANSACTIONS on FLUID MECHANICS, 2(3), pp: 90-100, 2008.

[6] Marsahall I., Zhao S., Papathanasopoulou P., Hoskins P., Yun Xu X., MRI and CFD studies of pulsatile flow in healthy and stenosed carotid bifurcation models, Journal of Biomechanics, 37, pp: 679-687, 2004.

[7] Rathish Kumar B.V., Yamaghuchi T., Liu H., Himeno R., A numerical study of an unsteady laminar flow in a doubly constricted 3D vessel, Int. J. Numer. Meth. Fluids, 38, pp: 1159-1176, 2002.

[8] Saa A.A., An experimental investigation of pulsatile flow through a smooth constriction, Experimental Thermal and Fluid Science, 17, pp: 309-318, 1998.

[9] Shahcheraghi N., Dwyer H.A., Cheer A.Y., Barakat A.I., Rutaganira T., Unsteady and Tree-Dimensional Simulation of Blood Flow in the Human Aortic Arch, Journal of Biomechanical Engineering, 124, pp: 378-387, 2002.

[10] Sherwin S.J., Blackburn H.M., Three-dimensional instabilities and transition of steady and pulsatile axisymmetric stenotic flows, J. Fluid Mech., 533, pp: 297-327.

[11] FLUENT 6.3 User's Guide, Fluent Incorporated, 2006. 